\title{
FITC-Labeled Alendronate as an In Vivo Bone pH Sensor
}

\author{
Yuzhou Li $\mathbb{D}^{1,2,3}$ Yiru Fu, ${ }^{1,2,3}$ He Zhang, ${ }^{1,2,3}$ Jinlin Song $\mathbb{D}^{1,2,3}$ and Sheng Yang $\mathbb{D}^{1,2,3}$ \\ ${ }^{1}$ College of Stomatology, Chongqing Medical University, Chongqing 401147, China \\ ${ }^{2}$ Chongqing Key Laboratory of Oral Diseases and Biomedical Sciences, Chongqing 401147, China \\ ${ }^{3}$ Chongqing Municipal Key Laboratory of Oral Biomedical Engineering of Higher Education, Chongqing 401147, China
}

Correspondence should be addressed to Jinlin Song; songjinlin@hospital.cqmu.edu.cn and Sheng Yang; ysdentist@hospital.cqmu.edu.cn

Received 20 January 2020; Revised 5 March 2020; Accepted 6 April 2020; Published 20 May 2020

Academic Editor: Sergio Murgia

Copyright @ 2020 Yuzhou Li et al. This is an open access article distributed under the Creative Commons Attribution License, which permits unrestricted use, distribution, and reproduction in any medium, provided the original work is properly cited.

\begin{abstract}
$\mathrm{pH}$ is a critical indicator of bone physiological function and disease status; however, noninvasive and real-time sensing of bone $\mathrm{pH}$ in vivo has been a challenge. Here, we synthesized a bone $\mathrm{pH}$ sensor by labeling alendronate with the $\mathrm{H}+$-sensitive dye fluorescein isothiocyanate (Aln-FITC). Aln-FITC showed selective affinity for hydroxyapatite (HAp) rather than other calcium materials. An in vivo biodistribution study showed that Aln-FITC can be rapidly and specifically delivered to rat bones after caudal vein injection, and the fluorescence lasted for at least $12 \mathrm{~h}$. The fluorescence intensity of Aln-FITC binding to HAp linearly decreased when the $\mathrm{pH}$ changed from 6 to 12. This finding was further confirmed on bone blocks and perfused bone when the $\mathrm{pH}$ changed from 6.8 to 7.4 , indicating unique $\mathrm{pH}$-responsive characteristics in the bone microenvironment. Aln-FITC was then preliminarily applied to evaluate the changes in bone $\mathrm{pH}$ in a nude mouse acidosis model. Our results demonstrated that Aln-FITC might have the potential for minimally invasive and real-time in vivo bone $\mathrm{pH}$ sensing in preclinical studies of bone healing, metabolism, and cancer mechanisms.
\end{abstract}

\section{Introduction}

$\mathrm{pH}$ plays an important role in bone homeostasis [1-4], and disruption of bone $\mathrm{pH}$ is associated with tumors, inflammation, fractures, and hypoxia $[1,2,4,5]$. Real-time sensing of bone $\mathrm{pH}$ can provide valuable information for diagnosis and research, but delivery of the sensor is challenged by hard and solid cortical bone [6]. Previous studies have tried to insert a pin-like microelectrode into the bone, which could report the $\mathrm{pH}$ value through a wired, external recorder $[7,8]$. Recently, a pH-responsive hydrogel-based implanted sensor with a metal pin was developed, where the $\mathrm{pH}$ data could be collected by a series of X-rays [9]. However, these sensors were all constrained by their expensive devices, low spatial resolution, and invasive surgery. To overcome these limitations, we assumed that a good bone $\mathrm{pH}$ sensor should be easy to fabricate, easy to deliver, and sensitive to real-time $\mathrm{pH}$ changes.

Fluorescent dyes are widely used in biology to sense organic targets such as proteins $[10,11]$ and nucleotides [12] and inorganic targets such as calcium [13], sodium
[14], and hydrogen ions $[15,16]$. Among these dyes, fluorescein isothiocyanate (FITC) has a H+-dependent emission spectrum over the range of $\mathrm{pH} 5-9$; thus, it is widely applied to sense the $\mathrm{pH}$ in biological conditions such as cells, soft tissues, and body fluids $[17,18]$. However, whether FITC can sense real-time changes in bone $\mathrm{pH}$ has not yet been studied.

The effective delivery of fluorescent sensors to the bone and maintenance of its concentration can also be challenging. Since the bone mainly comprises hydroxyapatite (HAp), molecules can be specifically targeted to the bone through modification with HAp affinity ligands, such as polyamino acids [19, 20], polypeptides [21], and phosphonates [22]. Alendronate, a typical phosphonate, can tightly bind to HAp through its two phosphate groups and has been broadly used in bone-targeting vehicles [23] and drugs [24]. In addition, alendronate can be retained in the bone for a long period of time [22]. Hence, modifying sensors with alendronate could reasonably enhance targeted delivery to the bone.

Herein, we introduce a bone $\mathrm{pH}$ sensor synthesized by alendronate and FITC, namely, Aln-FITC. The high 
reactivity of the isothiocyanate group (FITC) and amino group (alendronate) allowed the synthetic procedure to be simple and fast. The biocompatibility, biodistribution, and pH-responsive fluorescent characteristics of Aln-FITC were evaluated. The $\mathrm{pH}$-sensing ability of Aln-FITC was primarily tested in a metabolic acidosis animal model. To our knowledge, there have been few efforts regarding the use of fluorescent dyes to sense bone $\mathrm{pH}$ in vivo. We hypothesized that AlnFITC can work as a simple, safe, and effective bone $\mathrm{pH}$ sensor with future potential in preclinical studies for the in-depth understanding of changes in the bone microenvironment during healing, metabolic disease, and cancer development.

\section{Materials and Methods}

2.1. Ethics Statement. All experiments and procedures were performed in accordance with the appropriate guidelines of Chongqing Medical University. All operations for animals followed the guidelines of the Institutional Animal Care and Use Committee of China and were approved by the ethics committee of Chongqing Medical University Affiliated School and Hospital of Stomatology (2019073).

2.2. Synthesis of Aln-FITC. Fluorescein isothiocyanate isomer I ( $1.13 \mu \mathrm{mol}, \mathrm{F} 7250$, Sigma) was dissolved in DMSO and then mixed with $11.3 \mu \mathrm{mol}$ of alendronate dissolved in bicarbonate buffer, $\mathrm{pH} 9.0$. The volume was diluted to $1 \mathrm{ml}$ with distilled water, and the solution was incubated for $2 \mathrm{~h}$ at room temperature under constant mixing. To precipitate alendronate, $39.6 \mu \mathrm{mol}$ of $\mathrm{CaCl}_{2}$ was added, and the mixture was centrifuged (14,000 g, $10 \mathrm{~min})$. The precipitate was washed with $1 \mathrm{ml}$ of distilled water five times. To redissolve alendronate, $39.6 \mu \mathrm{mol}$ of EDTA was added to the precipitate. Then, $100 \mu \mathrm{l}$ of PBS was added until all precipitate dissolved, and the solution was shaken with ultrasonication for $30 \mathrm{~min}$. The final solution (referred to as Aln-FITC) was lyophilized, and an orange powder was obtained containing FITC-labeled alendronate and free alendronate, which was quantified and purified by liquid chromatography.

2.3. Characterization of Aln-FITC. Mass spectrometry (MS) spectra were obtained with $100 \%$ acetonitrile containing $0.1 \%$ formic acid in water at a flow rate of $0.3 \mathrm{ml} / \mathrm{min}$ using UP LC-MS (Shimadzu LC-MS 8060). Fourier transform infrared (FTIR) spectra were obtained between 400 and $4000 \mathrm{~cm}^{-1}$ with a resolution of $4 \mathrm{~cm}^{-1}$ using a Nicolet iS50 spectrometer (Thermo Fisher Scientific, USA) with the $\mathrm{KBr}$ disk method.

The absorption, excitation, and emission spectra of FITC, alendronate, and Aln-FITC were measured using a multiplate reader (EnSpire, USA). The absorption spectra were measured at wavelengths between $300 \mathrm{~nm}$ and $700 \mathrm{~nm}$. For the excitation spectra, samples were excited from 300 to $500 \mathrm{~nm}$ with a $10 \mathrm{~nm}$ step and measured at $530 \mathrm{~nm}$. For the emission spectra, samples were excited at $480 \mathrm{~nm}$ and measured in the range of 500 to $700 \mathrm{~nm}$ with a $10 \mathrm{~nm}$ step.

2.4. Cell Culture and Cytotoxicity Test. To acquire bone marrow stromal cells derived from rats (rBMSCs), two 6- to 8week-old male Sprague Dawley (SD) rats were sacrificed by cervical dislocation, and their femurs and tibias were carefully cleaned from the adherent soft tissue. The tip of each bone was removed with a rongeur, and the marrow was harvested by inserting a syringe needle ( 27 gauge) into one end of the bone and flushing with Dulbecco's modified Eagle's medium (DMEM, Gibco). The bone marrow cells were filtered through a $70 \mathrm{~mm}$ nylon mesh filter (BD Falcon, USA). Cells were plated into dishes $(d=10 \mathrm{~cm})$ in DMEM containing $15 \%$ FBS, $100 \mathrm{U} / \mathrm{ml}$ penicillin, and $100 \mathrm{U} / \mathrm{ml}$ streptomycin. Cultures were kept at $37^{\circ} \mathrm{C}$ in a humidified atmosphere containing $95 \%$ air and $5 \% \mathrm{CO}_{2}$. When the primary culture reached $90 \%$ confluency, the cells were treated with trypsin $(0.25 \% w / v)$ and EDTA $(0.02 \% w / v)$ for $2 \mathrm{~min}$ at room temperature and then subcultured in new dishes.

rBMSCs were cultured in 96-well plates (5,000 cells/well) for $24 \mathrm{~h}$ and treated with Aln-FITC over a range of concentrations ( 0 as the control, $0.1,0.5,1,5$, and $10 \mu \mathrm{M}$ ). After $24 \mathrm{~h}$, the rBMSCs were thoroughly washed to remove excess Aln-FITC, and cell growth medium (100 $\mu \mathrm{l} /$ well $)$ containing CCK-8 solution $(10 \mu \mathrm{l} /$ well, MCE) was added to each well, followed by incubation for $4 \mathrm{~h}$ at $37^{\circ} \mathrm{C}$. Then, the medium was transferred to a new 96-well plate to avoid any interference from intracellular Aln-FITC, and the absorbance at $450 \mathrm{~nm}$ was measured by a multiplate reader. For each concentration of Aln-FITC, the mean value was calculated from six samples.

2.5. In Vitro HAp and Bone Affinity of Aln-FITC. Aqueous suspensions of calcium species ( $\mathrm{HAp}, \mathrm{CaSO}_{4}$, and $\mathrm{CaC}_{2} \mathrm{O}_{4}$; all purchased from Macklin, China) $(5 \mathrm{mg} / \mathrm{ml})$ were incubated with Aln-FITC $(1 \mu \mathrm{M})$ for $2 \mathrm{~h}$. The unbound sensor in the supernatant was removed through centrifugation at $600 \mathrm{~g}$ for $5 \mathrm{~min}$, and the precipitate was washed with water under continuous agitation for $15 \mathrm{~min}$. This process of centrifugation and washing was repeated three times. Fluorescence analysis of the final aqueous product was performed using fluorescence microscopy.

Passage 4 rBMSCs plated at 10,000 cells/well in a 6-well plate were incubated in DMEM supplemented with $10 \%$ FBS and $100 \mathrm{U} / \mathrm{ml}$ penicillin/100 U/ml streptomycin for 2 days. Then, the proliferation medium was replaced with osteogenic medium containing DMEM with $50 \mu \mathrm{M}$ ascorbic acid 2-phosphate (Sigma), $10 \mathrm{nM}$ dexamethasone (Sigma), and $10 \mathrm{mM} \beta$-glycerol phosphate (Sigma). The cultures were then placed in an incubator at $37^{\circ} \mathrm{C}$ and $5 \% \mathrm{CO}_{2}$ for 21 days with media changes three times per week. At the end of the cultivation period, the media was removed, and the cell monolayer was washed with PBS and then incubated with DMEM containing Aln-FITC $(1 \mu \mathrm{M})$ for $2 \mathrm{~h}$. The media was removed and the cell monolayer was thoroughly washed three times with PBS, fixed with 4\% PFA for $15 \mathrm{~min}$, and washed with PBS $(3 \mathrm{x})$. The cells were then stained with Alizarin $\mathrm{S}(2 \%, \mathrm{pH} 4.2)$ for $5 \mathrm{~min}$ at room temperature. The supernatant was discarded, and the cell monolayer was washed with PBS (3x) and then imaged at excitation wavelengths of $530 \mathrm{~nm}$ (Alizarin S) and $488 \mathrm{~nm}$ (Aln-FITC).

2.6. In Vivo Bone Affinity and Toxicology of Aln-FITC. AlnFITC was intravenously injected into 3 male SD rats through the caudal vein at a dose of $8 \mu \mathrm{mol} / \mathrm{kg}$ body weight. The rats 
were sacrificed at $1 \mathrm{~h}, 4 \mathrm{~h}$, and $12 \mathrm{~h}$ postinjection. The heart, spleen, lung, liver, kidney, and femur were excised immediately and subsequently washed with saline three times for FITC fluorescence imaging on an in vivo imaging system (IVIS Lumina LT, PerkinElmer, Santa Clara, CA, USA). The results were quantitatively analyzed using Living Image software (PerkinElmer, USA).

After sacrifice, histological analyses of the liver, spleen, kidney, heart, and lung by H\&E staining were performed to assess the in vivo toxicology $12 \mathrm{~h}$ after injection of AlnFITC. An untreated SD male rat was used as the control.

2.7. pH-Responsive Characteristics of Aln-FITC. Aln-FITC was diluted to $1 \mu \mathrm{M}$ in $\mathrm{PBS}$ with $\mathrm{pH}$ values ranging from 2 to 14 . A similar experiment was performed with $1 \mu \mathrm{M}$ AlnFITC bound to HAp particles at $\mathrm{pH}$ values varying from 6 to 12 since a $\mathrm{pH}$ lower than 6 caused visible dissolution of HAp. The fluorescence intensity of each sample was measured with a multiplate reader. Thin bone blocks were dissected from the rat femurs as described in Section 2.6 and were also submerged in PBS with $\mathrm{pH}$ values from 6.8 to 7.4 and observed under a microscope (EVOS FL Auto). Excitation and emission wavelengths were adjusted according the reported maxima for Aln-FITC.

Aln-FITC was further intravenously injected into 3 male $\mathrm{SD}$ rats at a dose of $8 \mu \mathrm{mol} / \mathrm{kg}$ body weight. The donor mice were sacrificed after $12 \mathrm{~h}$. The femurs were excised immediately and subsequently perfused with saline for $1 \mathrm{~min}$, followed by perfusion with $1 \mathrm{x}$ PBS at four $\mathrm{pH}$ values $(\mathrm{pH} 6.8,7.0,7.2$, and 7.4). To avoid interference caused by the order of perfusion liquid or overwashing with Aln-FITC, the bones were perfused in random orders of PBS with different $\mathrm{pH}$ values. Then, the fluorescence intensity of Aln-FITC bound to the bone was measured on the in vivo imaging system.

2.8. In Vivo pH Sensing in a Metabolic Acidosis Model. AlnFITC was intravenously injected into 3 nude mice (male, 46 weeks old, $8 \mu \mathrm{mol} / \mathrm{kg}$ body weight). At $12 \mathrm{~h}$ postinjection, the mice were anesthetized by intraperitoneal injection of chloral hydrate and intravenously injected with 3\% lactic acid to simulate metabolic acidosis. After $10 \mathrm{~min}$, the mice were intravenously injected with $5 \%$ sodium bicarbonate for treatment. The fluorescence intensity was measured with the in vivo imaging system.

2.9. Statistical Analysis. All data are presented as the mean \pm standard deviation. The difference between two groups was analyzed by unpaired Student's $t$-test, and the difference among three or more groups was analyzed by one-way ANOVA and LSD as the post hoc test. In all comparisons, $P<0.05$ was set as the significant difference. All statistical analyses and graphs were processed in OriginPro 9.0 (OriginLab, USA).

\section{Results and Discussion}

3.1. Synthesis and Characterization of Aln-FITC. Aln-FITC was synthesized by conjugating the $\mathrm{pH}$-sensitive dye FITC with the bone-targeting ligand alendronate, as illustrated in Figure 1(a), and the final product was an orange powder.
To confirm the chemical structure, the final product was analyzed by FTIR (Figure 1(b)) and MS (Figure 1(c)). As shown in Figure 1(b), free FITC displayed a peak at $2015 \mathrm{~cm}^{-1}$ corresponding to the isothiocyanate group, and the free alendronate showed a characteristic peak at $1700 \mathrm{~cm}^{-1}$ assigned to the $\mathrm{N}-\mathrm{H}$ bending. After conjugation, both peaks disappeared, and a strong peak at $1733 \mathrm{~cm}^{-1}$ emerged, indicating the formation of thiohydantoin. At the same time, broad peaks ranging from 1300 to $750 \mathrm{~cm}^{-1}$ appeared in both alendronate and Aln-FITC, suggesting the presence of the remaining phosphate groups in the final product. Moreover, the MS confirmed the molecular weight of Aln-FITC as 636.85, which is consistent with the theoretical value of Aln-FITC, also indicating a successful synthesis.

The optical and fluorescent characteristics of Aln-FITC are shown in Figs S1-3. First, the absorption spectrum (Fig S1) showed a maximum absorbance peak at approximately $460 \mathrm{~nm}^{-1}$ in both the FITC and Aln-FITC spectra and no absorbance in the alendronate spectrum. In the fluorescence excitation spectrum (Fig S2) and the fluorescence emission spectrum (Fig S3), Aln-FITC showed a maximal excitation peak at $450-475 \mathrm{~nm}^{-1}$ and a maximal emission peak at approximately $525 \mathrm{~nm}^{-1}$, which is similar to the FITC results. Therefore, the optical and fluorescent parameters of AlnFITC were the same as those of FITC.

3.2. In Vitro and In Vivo Affinity of Aln-FITC to HAp and Bone. After incubation with Aln-FITC, HAp showed the highest intensity of green fluorescence (Figure 2(a)). Quantitative analysis revealed that the bonding ratio to HAp $(77.93 \pm 5.50 \%$, Figure $2(\mathrm{~b}))$ was threefolds higher than that to $\mathrm{CaSO}_{4}(18.44 \pm 4.59 \%, P<0.001)$ and fifteenfolds higher than that to $\mathrm{CaC}_{2} \mathrm{O}_{4}(4.66 \pm 1.82 \%, P<0.001)$. The average fluorescence intensity (Figure $2(\mathrm{c})$ ) on HAp $(138.1 \pm 26.3)$ was also higher than that on $\mathrm{CaSO}_{4}(56.1 \pm 8.6, P<0.001)$ and $\mathrm{CaC}_{2} \mathrm{O}_{4}(6.9 \pm 2.8, P<0.001)$. These results demonstrated the selective affinity of Aln-FITC towards HAp.

Fluorescence microscopy (Figure 2(d)) showed that the localization of Aln-FITC (green) basically overlapped with Alizarin S (red) in the differentiated rBMSCs, suggesting the selective affinity of Aln-FITC to calcified nodules compared with other cellular structures. This result demonstrated the selective targeting of Aln-FITC towards bone-like structures.

The in vivo targeting selectivity of Aln-FITC to the bone was also confirmed in Figure 2(e). After caudal intravenous injection of Aln-FITC, the bone showed the highest green fluorescence among the major organs, including the heart, spleen, lung, liver, and kidney. Quantitative analysis (Figure 2(f)) proved that $1 \mathrm{~h}$ after injection, the radiant efficiency in the bone increased to $(1.36 \pm 0.03) \times 10^{9}$ units, which was almost $66.3 \%$ of the maximal radiant efficiency, suggesting a fast accumulation in the bone after only $1 \mathrm{~h}$. Four hours after injection, the radiant efficiency in the bone reached its peak of $(2.05 \pm 0.07) \times 10^{9}$ units. At $12 \mathrm{~h}$ postinjection, the radiant efficiency remained at $(1.99 \pm 0.26) \times$ $10^{9}$ units, approximately $97.1 \%$ of the maximal radiant efficiency, indicating a long-lasting high concentration of AlnFITC in the bone. In other organs, the highest radiant 


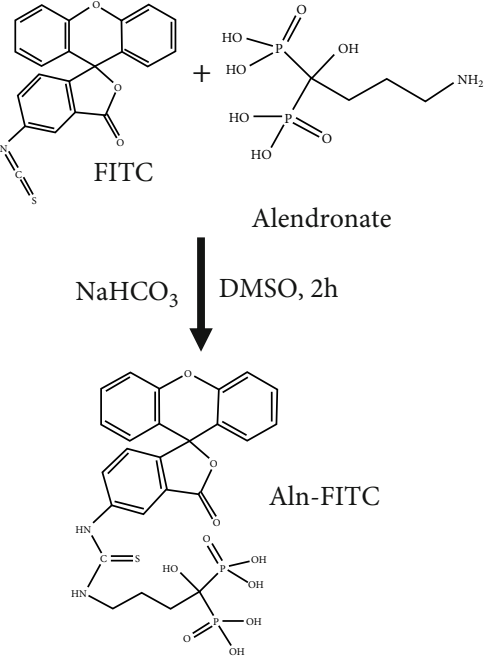

(a)

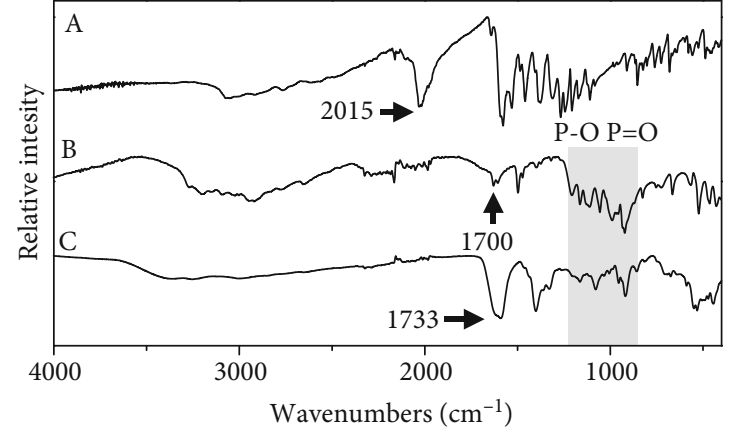

(b)

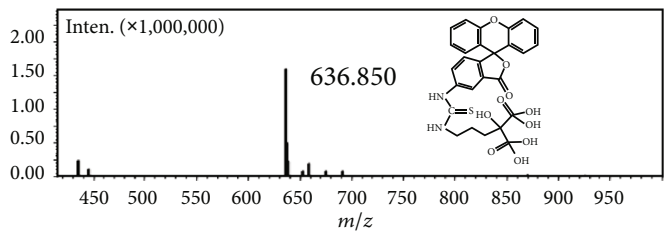

(c)

FIGURE 1: Synthesis and characterization of alendronate-FITC (Aln-FITC). (a) Schematic synthesis process of Aln-FITC. (b) FTIR spectra of FITC, alendronate, and Aln-FITC. (c) Mass spectrum of Aln-FITC.

efficiency appeared in the kidney $4 \mathrm{~h}$ after injection, which was $(2.46 \pm 0.03) \times 10^{8}$ units, suggesting that unbound AlnFITC was excreted by the kidneys. Therefore, these results indicated that Aln-FITC is a bone-targeted sensor suitable for long-term real-time sensing.

3.3. Biocompatibility of Aln-FITC. The cytotoxicity test of Aln-FITC in rBMSCs (Fig S4) exhibited no marked cytotoxicity when the concentration of Aln-FITC ranged from $0.1 \mu \mathrm{M}$ to $1 \mu \mathrm{M}$. However, the cytotoxicity became significant when the concentration increased to $5 \mu \mathrm{M}(0.90 \pm 0.06, P<$ $0.05)$ and $10 \mu \mathrm{M}(0.86 \pm 0.03, P<0.01)$. These results indicated that Aln-FITC had good biocompatibility when the concentration was no greater than $1 \mu \mathrm{M}$, which serves as a reference for further biological applications. The in vivo toxicology was also examined by the corresponding histological analyses of the major organs, including the liver, spleen, kidney, heart, and lung, with H\&E staining (Fig S5), suggesting no apparent histological toxicology of Aln-FITC at the dose of $8 \mu \mathrm{mol} / \mathrm{kg}$ body weight compared with the untreated control.

3.4. Unique pH-Responsive Characteristics of Aln-FITC on HAp and Bone. The pH-responsive characteristics of AlnFITC are shown in Figure 3. First, we studied the response curves of free Aln-FITC and Aln-FITC/HAp (Figure 3(a)). The fluorescence intensity of free Aln-FITC changed in the $\mathrm{pH}$ range of 2-10 in a pattern similar to many previous findings on FITC $[25,26]$. However, the $\mathrm{pH}$-responsive curve of
Aln-FITC/HAp left-shifted compared with that of FITC, resulting in a unique decreasing trend when the $\mathrm{pH}$ varied from 6 to 12 .

Then, we confirmed this phenomenon in bone blocks immersed in PBS at different $\mathrm{pH}$ values using fluorescence microscopy (Figure 3(b)). We found that the average fluorescence intensity linearly decreased $\left(R^{2}=0.95467\right)$ at $\mathrm{pH}$ values of $6.8 \quad(57.61 \pm 6.42), \quad 7.0 \quad(50.13 \pm 5.30), \quad 7.2$ (43.25 \pm 2.67$)$, and $7.4(31.78 \pm 6.12)$, and the loss of fluorescence was more obvious in the thin part of the bone blocks than in the thick part, indicating that the change started from the bone surface.

To establish a relationship between $\mathrm{pH}$ change and fluorescence intensity under the in vivo imaging systems, we analyzed the radiant efficiency of Aln-FITC binding to the bone under different $\mathrm{pH}$ values in a perfused bone model in Figure 3(c). When the $\mathrm{pH}$ increased from 6.8 to 7.4 , the radiant efficiency rapidly decreased. The calibration curve of the $\mathrm{pH}$-radiant efficiency in the bone (Figure $3(\mathrm{~d})$ ) showed that the radiant efficiency was $(4.2 \pm 0.31) \times 10^{9}$ units at $\mathrm{pH} 6.8$ and fell to $(1.1 \pm 0.34) \times 10^{9}$ units at $\mathrm{pH} 7.4$, decreasing in a linear manner $\left(R^{2}=0.96466\right)$. That is, in the $\mathrm{pH}$ range of 6.8-7.4, the relative radiant efficiency will decrease by $24.60 \%$ when the $\mathrm{pH}$ increases by 0.2 units as a preliminary calibration relationship to calculate the relative $\mathrm{pH}$ changes in the bone.

3.5. Preliminary Applications in a Metabolic Acidosis Model. We further tested the ability of Aln-FITC to sense bone $\mathrm{pH}$ 


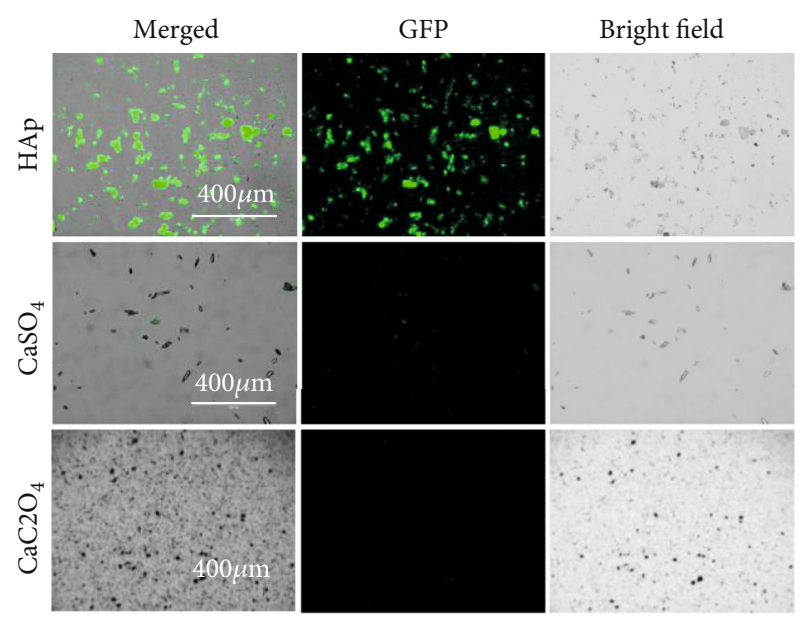

(a)

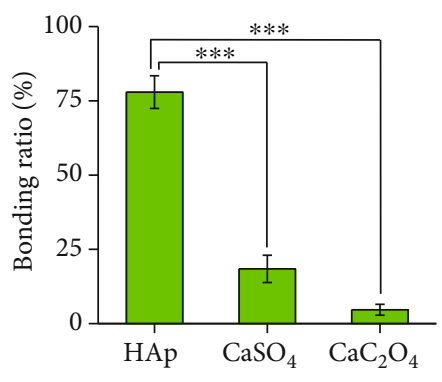

(b)

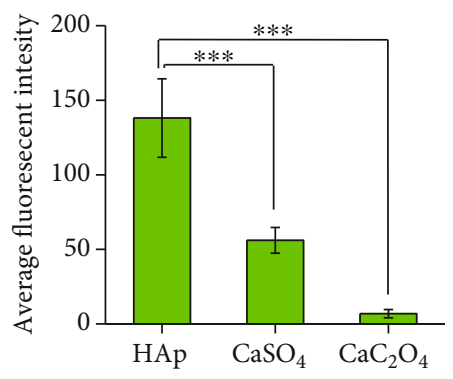

(c)
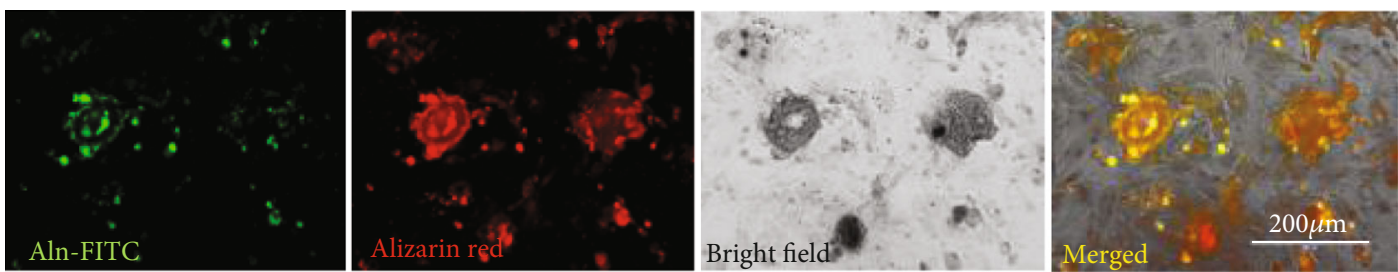

(d)
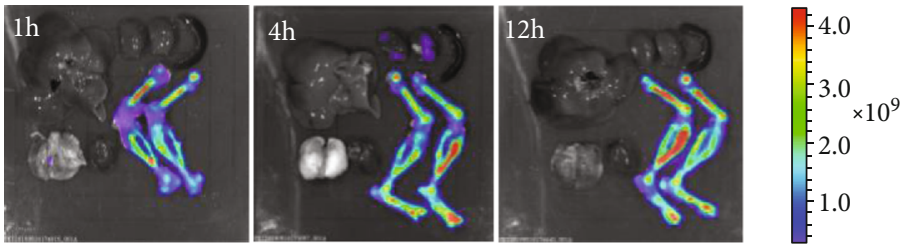

Radiant efficiency $\left(\mathrm{p} / \mathrm{sec} / \mathrm{cm}^{2} / \mathrm{sr}\right) /\left(\mu \mathrm{W} / \mathrm{cm}^{2}\right)$

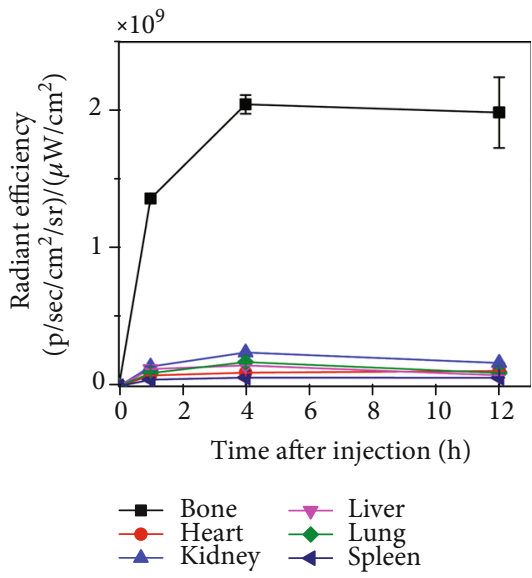

(e)

(f)

FIgURE 2: Selective affinity of Aln-FITC towards HAp and bone. (a) Fluorescence microscopy images of Aln-FITC binding to different calcium materials $\left(\mathrm{HAp}, \mathrm{CaSO}_{4}\right.$, and $\mathrm{CaC}_{2} \mathrm{O}_{4}$ ). Scale bar $=400 \mu \mathrm{m}$. (b, c) Quantified analysis of the fluorescence intensity of different calcium minerals. The bonding ratio was calculated as the ratio of positive fluorescent particle numbers to total particle numbers, while the average fluorescence intensity was measured from all positive fluorescent particles. (d) Fluorescence microscopy images of osteogenic differentiated rBMSCs stained with Aln-FITC and Alizarin S. Scale bar $=200 \mu \mathrm{m}$. (e) The in vivo biodistribution of Aln-FITC at $1 \mathrm{~h}, 4 \mathrm{~h}$, and $12 \mathrm{~h}$ postinjection in rat hearts, livers, spleens, lungs, kidneys, and bones. (f) Quantitative analysis of the time-radiant efficiency in different organs. $N=3$, mean $\pm \mathrm{SD}$.

changes in a metabolic acidosis model. In this preliminary study, since rat hairs may introduce fluorescent pollution and the penetration depth of Aln-FITC is relatively low, nude mice were chosen as the model animal due to their hairless skin and thinner soft tissue. Twelve hours after injection, Aln-FITC was distributed mainly in the skull, spine, limbs, and pelvic bone, as shown in Figure 4(a). After intravenous injection of $3 \%$ lactic acid to simulate metabolic acidosis, the radiant efficiency increased by approximately $56.06 \%$ in the skull bone, $36.35 \%$ in the pelvic bone, $11.06 \%$ in the spine, and $21.62 \%$ in the limb bones. After injection of $5 \%$ sodium bicarbonate for treatment, the radiant efficiency decreased by approximately $33.68 \%$ in the skull bone, $6.9 \%$ in the pelvic bone, $11.92 \%$ in the spine, and $13.45 \%$ in the 


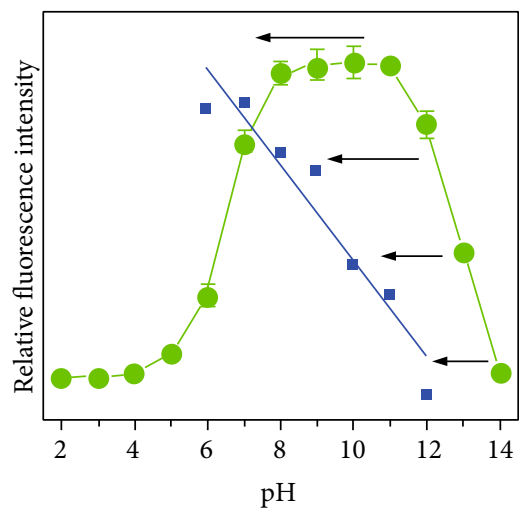

-Free Aln-FITC - Aln-FITC/HAp

(a)
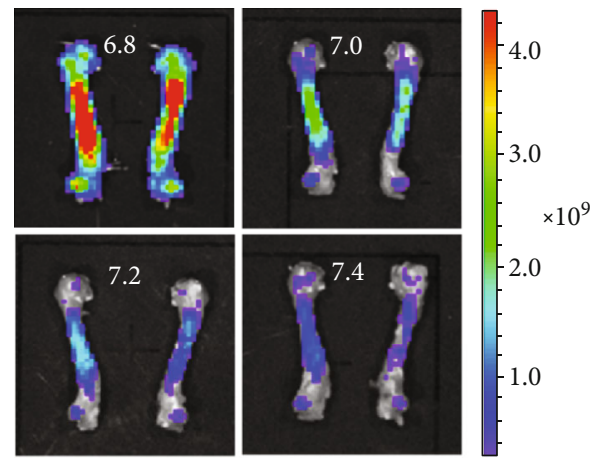

Radiant efficiency $\left(\mathrm{p} / \mathrm{sec} / \mathrm{cm}^{2} / \mathrm{sr}\right) /\left(\mu \mathrm{W} / \mathrm{cm}^{2}\right)$

(c)

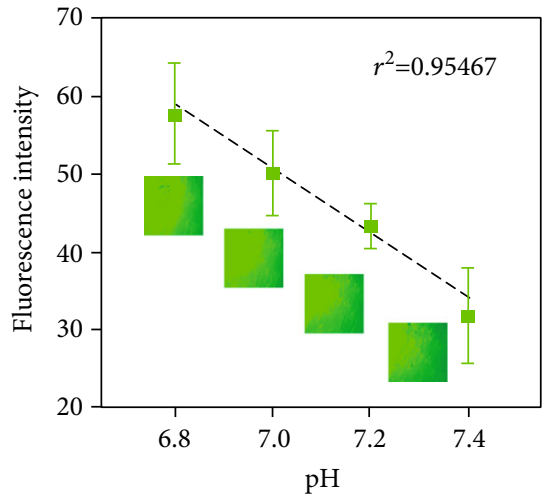

(b)

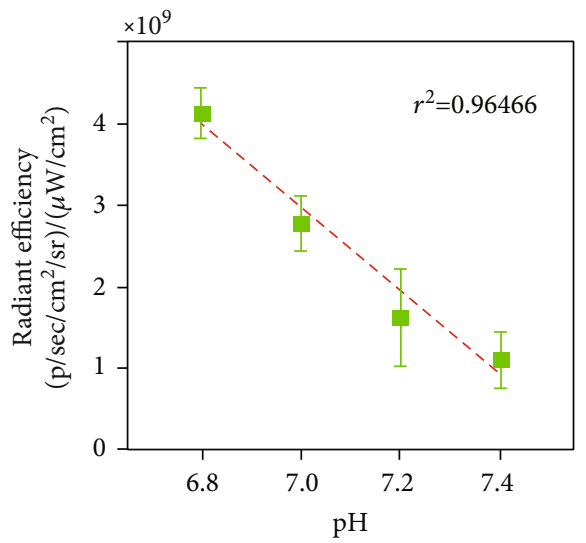

(d)

FIgURE 3: The $\mathrm{pH}$-responsive characteristics of Aln-FITC after binding to HAp and bone. (a) The pH-relative fluorescence intensity curve of free Aln-FITC (green) and Aln-FITC bonding to HAp (blue). Black arrows indicate the leftward shift after binding to HAp. (b) The fluorescence intensity in femur blocks decreased when the $\mathrm{pH}$ increased from 6.8 to 7.4. $N=3$, mean \pm SD. Representative fluorescence microscopy images of femur blocks bonded with Aln-FITC were affiliated with the data points. Note that the bottom right corner is the thin bone at the margin and the top left corner is the central thick bone. (c) The radiant efficiency of Aln-FITC in rat perfused bones over the $\mathrm{pH}$ range of 6.8-7.4. (d) The calibration curve of $\mathrm{pH}$-radiant efficiency showed that the radiant efficiency in perfused bone linearly decreased when the $\mathrm{pH}$ increased from 6.8 to 7.4. $N=3$, mean \pm SD.

limb bones (Figure 4(b)). It has been reported that the bone has an initial $\mathrm{pH}$ of approximately 7.4 [2], and so we calculated the relative $\mathrm{pH}$ changes according to the $\mathrm{pH}$ radiant efficiency relationship discovered in Section 3.4. Therefore, Aln-FITC indicated that the $\mathrm{pH}$ decreased by 0.12 in the skull, 0.08 in the pelvic bone, 0.02 in the spine, and 0.05 in the limb bones in the metabolic acidosis model (Figure 4(c)), and the $\mathrm{pH}$ value regained 0.11 units in the skull, 0.02 units in the pelvic bone, 0.02 units in the spine, and 0.04 units in the limb bones after treatment.

3.6. Limitations. The $\mathrm{pH}$ sensor in our study can be a useful tool in monitoring $\mathrm{pH}$ changes in basic and preclinical studies on bone physiology and diseases. However, we must point out that the maximal excitation peak of AlnFITC is at $450-475 \mathrm{~nm}$, and the maximal emission peak is at approximately $525 \mathrm{~nm}$, which implies that Aln-FITC has a low penetration depth and should be used in vitro or in vivo in preclinical animal models. We also found that animals with long hairs can easily bring background pollution to the fluorescence. Therefore, future studies should introduce novel dyes with higher wavelengths to increase the penetration depth and reduce background pollution from animal hairs [27]. For human applications, fluorescent sensors should be constructed in the near infrared range, which is called the diagnostic window [28].

In our study, the fluorescence intensities in different bones in the same state were different due to the different bone masses and different Aln-FITC concentrations, which makes it difficult to determine the absolute $\mathrm{pH}$ value. Therefore, we chose to calculate relative $\mathrm{pH}$ changes according to the relative fluorescence intensity changes at a single point. Previous studies have tried to introduce a reference indicator to avoid this problem by using $\mathrm{pH}$-insensitive dyes such as AuNCs and rhodamine [26, 29]. Combining a two-sensor system and bone-targeting ligand is challenging and shall be investigated in the future. In addition, compared with fluorescence intensity, novel fluorescence techniques such 

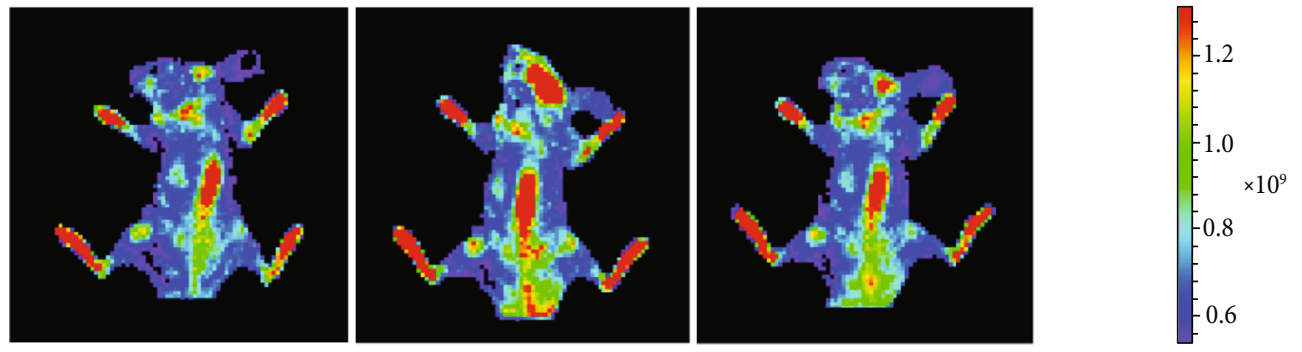

Radiant efficiency $\left(\mathrm{p} / \mathrm{sec} / \mathrm{cm}^{2} / \mathrm{sr}\right) /\left(\mu \mathrm{W} / \mathrm{cm}^{2}\right)$

(a)

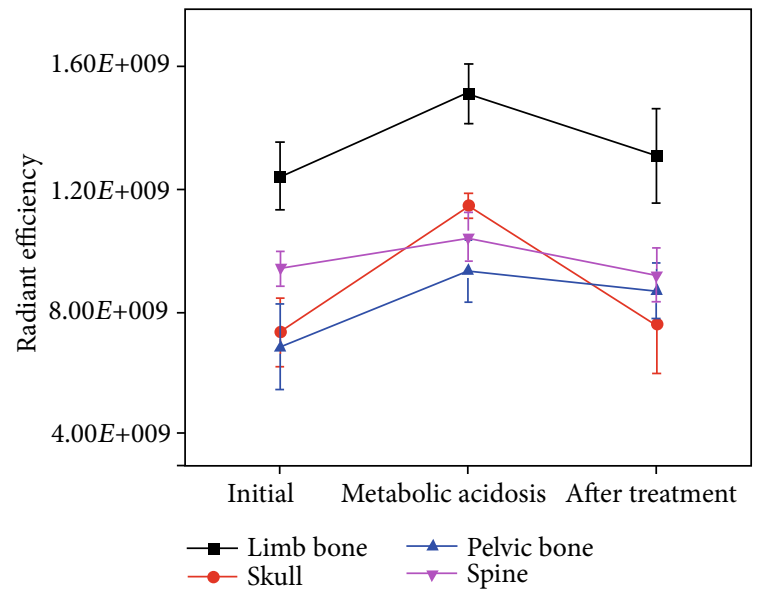

(b)

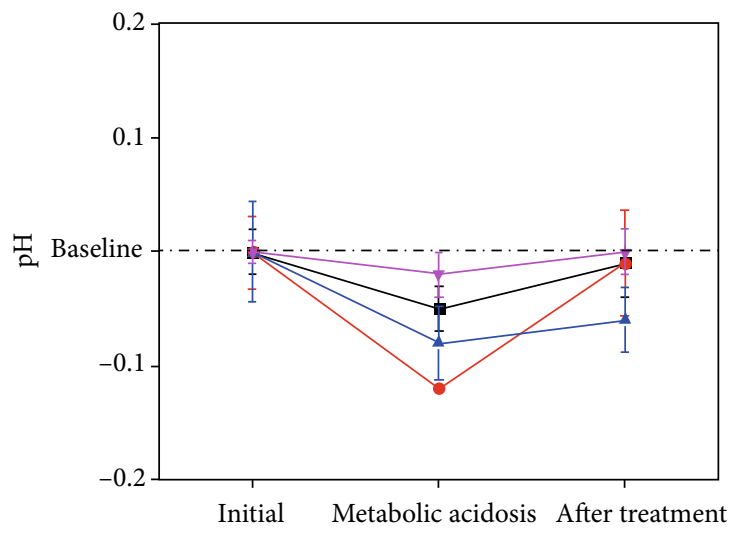

(c)

FIGURE 4: Application of Aln-FITC in sensing pH changes in a metabolic acidosis model. (a) In vivo fluorescent images of Aln-FITC in nude mice during three different states: the normal state, metabolic acidosis state, and alkaline drug treatment state. (b, c) Quantitative calculation of relative radiant efficiency and corresponding $\mathrm{pH}$ change of the skull (red), limbs (black), and pelvis (blue) under the three different conditions.

as fluorescein lifetime measurement $[30,31]$ showed better accuracy and specificity in in vivo $\mathrm{pH}$ sensing. Future studies should introduce fluorescence lifetime measurements to step into a broader application of bone $\mathrm{pH}$ sensors.

\section{Conclusion}

In this study, we developed a bone $\mathrm{pH}$ sensor (Aln-FITC) suitable for preclinical applications to monitor $\mathrm{pH}$ changes during bone healing, metabolic disease, and cancer. AlnFITC can be applied in rodents at a biologically safe concentration, avoiding the risk of surgical wound infection in previous $\mathrm{pH}$ detection methods. Aln-FITC has excellent bonetargeting ability and good $\mathrm{pH}$ sensitivity in the $\mathrm{pH}$ range of 6.8-7.4, making it suitable for detecting $\mathrm{pH}$ changes in ex vivo or in vivo nude mouse models. However, Aln-FITC is limited by the short wavelength and quantification difficulties in the absolute $\mathrm{pH}$ value without an internal reference, which shall be addressed by using a two-sensor system inside the diagnostic window and novel fluorescence techniques such as fluorescence lifetime imaging in future studies.

\section{Data Availability}

All data generated or analyzed during this study are included in this article and Supplementary Materials.

\section{Conflicts of Interest}

The authors declare no conflicts of interest. The authors declare no potential conflicts of interest with respect to the authorship and/or publication of this article.

\section{Authors' Contributions}

Conceptualization was handled by Yuzhou Li and Sheng Yang, formal analysis was handled by Yuzhou Li and Yiru $\mathrm{Fu}$, funding acquisition was handled by Jinlin Song and Sheng Yang, investigation was handled by Yiru Fu, methodology was handled by Yuzhou Li and He Zhang, supervision was handled by Jinlin Song and Sheng Yang, visualization was handled by Yuzhou Li and Yiru Fu, writing of the original draft was handled by Yuzhou Li and Yiru Fu, and review and editing of the manuscript was handled by He Zhang, Jinlin Song, and Sheng Yang. All authors have read and agreed to the published version of the manuscript. Yuzhou Li and Yiru Fu contributed equally to this work.

\section{Acknowledgments}

This work was supported by the National Natural Science Foundation of China (grant numbers 81901057 and 81500894), the General Program of China Postdoctoral Science 
Foundation (grant number 2017M620417), the Natural Science Foundation of Chongqing (grant numbers cstc2019jcyjmsxmX0366, cstc2017jcyjBX0019, and cstc2019jcyjbshX0005), and the Program for Innovation Team Building at Institutions of Higher Education in Chongqing in 2016 (CXTDG201602006).

\section{Supplementary Materials}

Figure S1: UV-vis spectra of FITC (A), Aln-FITC (B), and alendronate $(\mathrm{C})$. Figure S2: excitation spectra of FITC (A), Aln-FITC (B), and alendronate (C). Figure S3: emission spectra of FITC (A), Aln-FITC (B), and alendronate (C). Figure S4: cell viability of rBMSCs treated with Aln-FITC at different concentrations $(0 \mu \mathrm{M}, 0.1 \mu \mathrm{M}, 0.5 \mu \mathrm{M}, 1 \mu \mathrm{M}, 5 \mu \mathrm{M}$, and $10 \mu \mathrm{M})$ for $24 \mathrm{~h}, n=6$, mean \pm SD. Figure S5: histological analysis of major organs, including the liver, spleen, kidney, heart, and lung, by H\&E staining (scale bar $=50 \mu \mathrm{m}$ ). (Supplementary Materials)

\section{References}

[1] T. R. Arnett, "Extracellular pH regulates bone cell function," The Journal of Nutrition, vol. 138, pp. 415s-418s, 2008.

[2] D. A. Chakkalakal, A. A. Mashoof, J. Novak, B. S. Strates, and M. H. McGuire, "Mineralization and $\mathrm{pH}$ relationships in healing skeletal defects grafted with demineralized bone matrix," Journal of Biomedical Materials Research, vol. 28, no. 12, pp. 1439-1443, 1994.

[3] A. M. Galow, A. Rebl, D. Koczan, S. M. Bonk, W. Baumann, and J. Gimsa, "Increased osteoblast viability at alkaline $\mathrm{pH}$ in vitro provides a new perspective on bone regeneration," Biochemistry and Biophysics Reports, vol. 10, pp. 17-25, 2017.

[4] D. H. Kohn, M. Sarmadi, J. I. Helman, and P. H. Krebsbach, "Effects of $\mathrm{pH}$ on human bone marrow stromal cells in vitro: implications for tissue engineering of bone," Journal of Biomedical Materials Research, vol. 60, no. 2, pp. 292-299, 2002.

[5] S. Huang, Y. Tang, X. Peng et al., "Acidic extracellular $\mathrm{pH}$ promotes prostate cancer bone metastasis by enhancing PC-3 stem cell characteristics, cell invasiveness and VEGF-induced vasculogenesis of BM-EPCs," Oncology Reports, vol. 36, no. 4, pp. 2025-2032, 2016.

[6] G. R. Martin and R. K. Jain, "Noninvasive measurement of interstitial $\mathrm{pH}$ profiles in normal and neoplastic tissue using fluorescence ratio imaging microscopy," Cancer Research, vol. 54, no. 21, pp. 5670-5674, 1994.

[7] B. E. Beck-Broichsitter, D. W. Christofzik, F. Daschner et al., "Endocultivation: metabolism during heterotopic osteoinduction in vivo-monitoring with fiber optic detection devices," Tissue Engineering Part C: Methods, vol. 18, no. 10, pp. 740746, 2012.

[8] Y. Hazehara-Kunitomo, E. Hara, M. Ono et al., "Acidic preconditioning enhances the stem cell phenotype of human bone marrow stem/progenitor cells," International Journal of Molecular Sciences, vol. 20, no. 5, p. 1097, 2019.

[9] M. Arifuzzaman, P. W. Millhouse, Y. Raval et al., "An implanted $\mathrm{pH}$ sensor read using radiography," The Analyst, vol. 144, no. 9, pp. 2984-2993, 2019.

[10] P. J. Cranfill, B. R. Sell, M. A. Baird et al., "Quantitative assessment of fluorescent proteins," Nature Methods, vol. 13, no. 7, pp. 557-562, 2016.
[11] G. J. Kremers, S. G. Gilbert, P. J. Cranfill, M. W. Davidson, and D. W. Piston, "Fluorescent proteins at a glance," Journal of Cell Science, vol. 124, Part 2, pp. 157-160, 2011.

[12] H. Li, X. Fang, H. Cao, and J. Kong, "Paper-based fluorescence resonance energy transfer assay for directly detecting nucleic acids and proteins," Biosensors \& Bioelectronics, vol. 80, pp. 79-83, 2016.

[13] H. Ogasawara, M. Grzybowski, R. Hosokawa, Y. Sato, M. Taki, and S. Yamaguchi, "A far-red fluorescent probe based on a phospha-fluorescein scaffold for cytosolic calcium imaging," Chemical Communications, vol. 54, no. 3, pp. 299-302, 2018.

[14] A. Thien, J. X. Han, K. Kumar et al., "Investigation of the usefulness of fluorescein sodium fluorescence in stereotactic brain biopsy," Acta Neurochirurgica, vol. 160, no. 2, pp. 317-324, 2018.

[15] J. Qi, D. Liu, X. Liu et al., "Fluorescent pH sensors for broadrange $\mathrm{pH}$ measurement based on a single fluorophore," Analytical Chemistry, vol. 87, no. 12, pp. 5897-5904, 2015.

[16] D. Aigner, S. M. Borisov, F. J. Fernandez, J. F. Fernandez Sanchez, R. Saf, and I. Klimant, "New fluorescent pH sensors based on covalently linkable PET rhodamines," Talanta, vol. 99, pp. 194-201, 2012.

[17] J. W. Chen, C. M. Chen, and C. C. Chang, "A fluorescent pH probe for acidic organelles in living cells," Organic \& Biomolecular Chemistry, vol. 15, no. 37, pp. 7936-7943, 2017.

[18] R. Ohgaki, Y. Teramura, D. Hayashi et al., "Ratiometric fluorescence imaging of cell surface $\mathrm{pH}$ by poly (ethylene glycol)phospholipid conjugated with fluorescein isothiocyanate," Scientific Reports, vol. 7, no. 1, article 17484, 2017.

[19] O. H. Jeon and J. Elisseeff, "Orthopedic tissue regeneration: cells, scaffolds, and small molecules," Drug Delivery and Translational Research, vol. 6, no. 2, pp. 105-120, 2016.

[20] Y. R. Shih, Y. Hwang, A. Phadke et al., "Calcium phosphatebearing matrices induce osteogenic differentiation of stem cells through adenosine signaling," Proceedings of the National Academy of Sciences of the United States of America, vol. 111, no. 3, pp. 990-995, 2014.

[21] Y. Sun, X. Ye, M. Cai et al., "Osteoblast-targeting-peptide modified nanoparticle for siRNA/microRNA delivery," ACS Nano, vol. 10, no. 6, pp. 5759-5768, 2016.

[22] D. A. Ossipov, "Bisphosphonate-modified biomaterials for drug delivery and bone tissue engineering," Expert Opinion on Drug Delivery, vol. 12, no. 9, pp. 1443-1458, 2015.

[23] Q. Chen, C. Zheng, Y. Li et al., "Bone targeted delivery of SDF1 via alendronate functionalized nanoparticles in guiding stem cell migration," ACS Applied Materials \& Interfaces, vol. 10, no. 28, pp. 23700-23710, 2018.

[24] Z. Xie, G. Liu, P. Tang et al., "Bone-targeted methotrexatealendronate conjugate inhibits osteoclastogenesis in vitro and prevents bone loss and inflammation of collagen-induced arthritis in vivo," Drug Delivery, vol. 25, no. 1, pp. 187-197, 2018.

[25] K. Thompson, M. J. Rogers, F. P. Coxon, and J. C. Crockett, "Cytosolic entry of bisphosphonate drugs requires acidification of vesicles after fluid-phase endocytosis," Molecular Pharmacology, vol. 69, no. 5, pp. 1624-1632, 2006.

[26] L. Wang, Y. Zhou, Y. Zhang et al., "A novel cell-penetrating Janus nanoprobe for ratiometric fluorescence detection of $\mathrm{pH}$ in living cells," Talanta, vol. 209, p. 120436, 2020.

[27] J. R. Mansfield, C. C. Hoyt, P. J. Miller, and R. M. Levenson, "Distinguished photons: increased contrast with multispectral 
in vivo fluorescence imaging," BioTechniques, vol. 39, no. 6S, pp. S33-S37, 2005.

[28] A. Sakudo, "Near-infrared spectroscopy for medical applications: current status and future perspectives," Clinica Chimica Acta, vol. 455, pp. 181-188, 2016.

[29] Y. Yang, M. Xia, H. Zhao, S. Zhang, and X. Zhang, "A cellsurface-specific ratiometric fluorescent probe for extracellular pH sensing with solid-state fluorophore," ACS Sensors, vol. 3, no. 11, pp. 2278-2285, 2018.

[30] S. Schreml, R. J. Meier, O. S. Wolfbeis, M. Landthaler, R. M. Szeimies, and P. Babilas, "2D luminescence imaging of $\mathrm{pH}$ in vivo," Proceedings of the National Academy of Sciences of the United States of America, vol. 108, no. 6, pp. 2432-2437, 2011.

[31] R. Niesner, B. Peker, P. Schlüsche et al., "3D-resolved investigation of the $\mathrm{pH}$ gradient in artificial skin constructs by means of fluorescence lifetime imaging," Pharmaceutical Research, vol. 22, no. 7, pp. 1079-1087, 2005. 